\title{
669 LACTATE UPTAKE THROUGH MCT11, A NOVEL MONOCARBOXYLATE TRANSPORTER, ENFORCES DYSFUNCTION IN TERMINALLY EXHAUSTED T CELLS
}

Ronal Peralta*, Greg Delgoffe. University of Pittsburgh, Pittsburgh, PA, United States

Background Upon infiltration into tumors, T cells experiencing persistent antigen stimulation progressively differentiate into a state of dysfunction, known as exhaustion. Exhausted $\mathrm{T}$ cells are characterized by the sustained upregulation of co-inhibitory molecules and reduced effector cytokine production. Additionally, exhausted $\mathrm{T}$ cells exist in a state of metabolic dysfunction in the tumor microenvironment (TME), due to disrupted mitochondrial biogenesis, hypoxia and lack of metabolites. Highly glycolytic tumor and stromal cells outcompete $\mathrm{T}$ cells for glucose, and secrete lactate into the TME, acidifying the extracellular space. Recent studies have shown lactate can be metabolized by tumor infiltrating Tregs and macrophages. We hypothesized that CD8 + tumor-infiltrating lymphocytes (TIL) may also take up lactate as an alternative carbon source to meet their metabolic demands.

Methods For lactate uptake experiments, B16 melanoma single cell suspensions from $\mathrm{B} 6$ mice were loaded with the $\mathrm{pH}$ sensitive dye pHrodo, then pulsed with $5 \mu \mathrm{M}$ lactic acid. MCT11 KO OT-I T cells were generated via transfection of Slc16a11 sgRNA-Cas9 ribonucleoprotein complexes, and adoptively transferred into B16-OVA bearing mice.

Results RNA sequencing and flow cytometry data from CD8+ $\mathrm{T}$ cell subsets in the TME revealed MCT11 (encoded by Slc16a11), a monocarboxylate transporter (MCT) only recently discovered, to be highly and uniquely expressed in terminally exhausted $\mathrm{T}$ cells (Tex). As lactate is an abundant monocarboxylate in tumors, we asked whether MCT11 supports lactate uptake into Tex cells. Antibody blockade of MCT11 resulted in reduced lactic acid uptake, but whether lactic acid promoted or inhibited effector function. Intriguingly, overexpression of MCT11 in OT-I T cells adoptively transferred into B16-OVA bearing mice resulted in accelerated exhaustion: increased co-inhibitory marker expression and decreased TNFa and IFN production. Conversely, knockdown of MCT11 in the same model resulted in decreased co-inhibitory marker expression and increased TNFa and IFN production. Further, MCT11 KO OT-I T cells used therapeutically had decreased tumor burden over mice treated with control OT-I T cells. As MCT11's uptake function was blocked with an antibody, we also used the antibody therapeutically, revealing that singleagent MCT11 antibody treatment reduced tumor burden and increased survival in B16 melanoma bearing mice.

Conclusions Our data support a model where exhausted CD8 $+\mathrm{T}$ cells upregulate MCT11, which renders them sensitive to toxic lactic acid in the TME. Our data suggest MCT11 could be deleted on therapeutic $\mathrm{T}$ cells or blocked using an antibody on endogenous $\mathrm{T}$ cells to render exhausted $\mathrm{T}$ cells impervious to lactic acid such and promote tumor eradication.

http://dx.doi.org/10.1136/jitc-2021-SITC2021.669 